Abanico Veterinario. Enero-Diciembre 2020; 10:1-18. http://dx.doi.org/10.21929/abavet2020.38

Artículo Original. Recibido: 27/04/2020. Aceptado: 26/11/2020. Publicado: 22/12/2020. Clave:2020-35.

\title{
Tratamiento estacional con amitraz contra Varroa destructor y sus efectos en colonias de Apis mellifera
}

Seasonal treatment with amitraz against Varroa destructor and its effects in honey bee colonies

\section{Maya-Martínez Omar ${ }^{1}$ ID, Medina-Flores Carlos ${ }^{\star 2}$ ID, Aquino-Pérez Gildardo ${ }^{1 \text { ID, }}$ Olmos-Oropeza Genaro' ID, López-Carlos Marco' ${ }^{2}$ ID}

${ }^{1}$ Colegio de Postgraduados Campus Salinas de Hidalgo, San Luis Potosí, México. ${ }^{2}$ Unidad Académica de Medicina Veterinaria y Zootecnia, Universidad Autónoma de Zacatecas, México. *Autor responsable y de correspondencia: Medina-Flores Carlos. Carretera Panamericana Km 31.5 tramo Zacatecas-Fresnillo, El Cordovel Gral Enrique Estrada, Zacatecas, México, C.P. 98500. omarmaya_m@hotmail.com, carlosmedina@uaz.edu.mx, jaquino@colpos.mx,olmosg@colpos.mx, lopcarmarco@uaz.edu.mx

\section{RESUMEN}

Se determinó el efecto del tratamiento estacional con amitraz contra Varroa destructor sobre la población y reservas alimenticias de colonias de abejas melíferas durante las cuatro estaciones del año en el altiplano central de México. Se utilizaron 48 colonias con reinas hermanas, homogéneas en población, reservas de alimentos y niveles de Varroa. 12 colonias recibieron tratamiento en verano, 12 en invierno, 12 en verano e invierno y 12 no fueron tratadas. Los niveles de $V$. destructor en abejas adultas, en cría y la caída de ácaros en el piso de las colmenas se determinaron durante un año. También se evaluó la población de abejas, áreas de cría, miel, polen y peso de las colonias. Hubo diferencias en los niveles de Varroa entre tratamientos $(\mathrm{P}<0.05)$. Finalizando el experimento (primavera), el nivel de infestación en colonias tratadas en verano $(602 \pm 114)$ y no tratadas $(416 \pm 86)$ fueron superiores $(P=0.0002)$ que en las tratadas en verano e invierno $(109 \pm 50)$ o solo en invierno $(100 \pm 42)$, entre las cuales no hubo diferencias. Sin embargo, no hubo efecto significativo de los tratamientos sobre la población, reservas de alimentos y peso de las colonias. El tratamiento invernal fue suficiente para controlar Varroa en colonias del altiplano central de México.

Palabras clave: Varroa destructor, abejas melíferas, amitraz, población de abejas, reservas de alimento.

\section{ABSTRACT}

The objective of this work was to determine the amitraz treatment effect against Varroa destructor on the population and food reserves of honeybee colonies, during the four seasons of the year in Mexico's central high plateau. 48 colonies with similar sister queens, homogeneous populations, food reserves, and Varroa infestation levels were used.12 colonies received acaricidal treatment in the summer, 12 in winter, 12 in summer and winter, and 12 were untreated. The Varroa infestation levels were determined in adult bees, worker brood, and by mites on the hives' floor for one year. The adult bee population, capped brood area, honey, pollen, and colony weight were also evaluated. There were statistical differences $(\mathrm{P}<0.05)$ between the Varroa levels on treatments. Ending the experiment (spring) the infestation level in colonies treated in summer $(602 \pm 114)$ and not treated $(416 \pm 86)$ were higher $(P=0.0002)$ than those treated in summer and winter $(109 \pm 50)$, or only in winter $(100 \pm 42)$, between which, there were no statistical differences. However, there were no significant effects of the treatments on population bees, food stores, and weight. The winter treatment was sufficient to control Varroa infestation in colonies located in Mexico's central high plateau.

Keywords: Varroa destructor, honey bees, amitraz, bee population, food stores. 


\section{INTRODUCCIÓN}

El ácaro Varroa destructor (Acari: Varroidae) (Anderson y Trueman, 2000) causante de la varroosis, es el problema sanitario número uno para la apicultura a nivel mundial (Nazzi y Le Conte, 2016). Esto se debe a que su distribución es generalizada, afecta a la cría y abejas adultas, transmite y predispone a la presencia de enfermedades bacterianas, virales y fungales (Martin et al., 2010; vanEngelsdorp y Maixner, 2010; Ryabov et al., 2017), reduce el periodo de vida de las abejas (Dainat et al., 2012), el tamaño poblacional y la producción de miel de las colonias (Arechavaleta y GuzmánNovoa, 2000; Medina-Flores et al., 2011) y es considerado uno de los principales factores asociados a la alta pérdida anual de colonias (Marie-Pierre et al., 2010; Guzmán-Novoa et al., 2010).

Además de los problemas ya mencionados por Varroa a las colonias y la industria apícola, la aplicación de los acaricidas utilizados para su control, representan otro problema ya que en general todos han mostrado efectos adversos para las abejas, más aun si estos no son aplicados de forma correcta. Los productos sintéticos (fluvalinato, flumetrina y amitraz) han sido los más efectivos, particularmente el amitraz el cual ha mostrado ser menos residual y tóxico para las abejas (Gashout et al., 2018) y tener menos problemas de resistencia en la región del altiplano central de México (Rodríguez-Dehaibes et al., 2011). Sin embargo, estos acaricidas pueden afectar el desarrollo de reinas y zánganos, la habilidad de aprendizaje y la postura de la reina (Berry et al., 2013) y provocar el desarrollo de resistencia del ácaro (Martínez-Puc y Medina-Medina, 2011; Kamler et al., 2016). Los ácidos orgánicos, como el fórmico reduce la memoria Gashout et al., 2020), y el ácido oxálico afecta la longevidad y sobrevivencia de obreras y la cría (Schneider et al., 2012), mientras que los monoterpenos constituyentes de los aceites esenciales derivados de plantas, pueden tener efectos tóxicos y reducir la inmunidad humoral (Boncristiani et al., 2012). La eficiencia acaricida de los ácidos orgánicos y los extractos vegetales es variable y dependiente del aplicador y su posición en la colmena, de la humedad y temperatura ambiental, del tamaño de la colmena, de la presencia de cría, entre otros factores (Pietropaoli y Formato, 2018).

Para controlar las poblaciones de ácaros en las colonias y al mismo tiempo reducir el uso de acaricidas, es necesario identificar la época oportuna para aplicar los tratamientos; esto contribuirá a reducir los efectos negativos de los acaricidas antes mencionados, así como la presión de selección de ácaros resistentes, los riesgos de contaminación de los productos de las colonias, el costo de producción a los apicultores debido a la aplicación innecesaria de tratamientos y evitaría relajar la presión de selección para la resistencia a los ácaros (Delaplane y Hood, 1997; 1999; Delaplane, 1998; Caron, 1999; González-Cabrera et al., 2016). Sin embargo, el desarrollo 
poblacional del ácaro difiere regionalmente debido a la variación del período de cría en las colonias y sus efectos sobre la dinámica poblacional del ácaro (Delaplane, 1998; Caron, 1999). En consecuencia, el umbral de tratamiento debe ser determinado en regiones específicas. Por lo anterior, el objetivo del presente estudio fue determinar el efecto del tratamiento a base de amitraz aplicado en verano, invierno y verano e invierno sobre los niveles de infestación por Varroa destructor y las condiciones poblacionales y alimenticias de colonias de abejas melíferas bajo condiciones del altiplano central de México.

\section{MATERIAL Y MÉTODOS}

Ubicación. El apiario experimental se ubicó en Jalpa, Zacatecas, México, a $21^{\circ} 38^{\prime} \mathrm{N}$ y $100^{\circ} 51^{\prime} \mathrm{O}$, y $1,380 \mathrm{msnm}$. El área de estudio presenta una vegetación de tipo selva baja caducifolia y un clima semiseco semicálido. La temperatura media anual es de $21.2^{\circ} \mathrm{C}$ y una precipitación media anual de $700 \mathrm{~mm}$ (INEGI, 2019).

Colonias experimentales y aplicación de los tratamientos acaricidas. De una población de 200 colonias de abejas melíferas alojadas en colmenas tipo Langstroth, se seleccionaron 48 con condiciones similares. En promedio, las colonias experimentales estaban conformadas con ocho panales cubiertos con abejas, de los cuales cinco panales contenían cría operculada, dos panales con miel y uno con polen y un nivel promedio de infestación de $V$. destructor en abejas adultas de $5.3 \pm 0.36 \%$. Las colonias fueron establecidas en un solo apiario y sus reinas fueron remplazadas por reinas hermanas de la misma generación y origen.

Se formaron cuatro grupos experimentales, cada uno integrado con 12 colonias de abejas. Las colonias del primer grupo recibieron tratamiento contra Varroa en el verano (los días 10, 17, 24 y 31 de julio de 2016), las colonias del segundo grupo recibieron tratamiento durante el invierno (19, 26 de enero, 2 y 9 de febrero de 2017), el grupo tres recibió doble tratamiento, uno de ellos en verano y otro en el invierno (en las mismas fechas antes mencionadas) y las colonias del grupo cuatro no recibieron tratamiento durante todo el experimento. El propósito de aplicar doble tratamiento acaricida (grupo: verano e invierno) fue provocar niveles de infestación por Varroa más bajos que en las colonias tratadas solo en una estación del año y hacer comparaciones respecto a las condiciones poblacionales y de reservas alimenticias de las colonias, además, algunos apicultores utilizan dicho protocolo lo cual probablemente sea innecesario y adverso para las colonias. Generar esta información permite tomar decisiones sobre el número de tratamientos y época de aplicación más conveniente para el control de Varroa.

Los tratamientos contra Varroa consistieron en aplicar semanalmente y durante cuatro ocasiones $10 \mathrm{ml}$ de amitraz (Taktic $\AA$ ) al $1.25 \%$ en una toalla absorbente (Scott ${ }^{\circledR}$ ) de 28 
x $6.5 \mathrm{~cm}$ sobre los cabezales de los bastidores de la cámara de cría. El uso de amitraz en dichas preparaciones se basa en previas investigaciones realizadas por Lupo y Gerling (1990), Smodiš et al. (2011) y Gregorc y Planinc (2012) y en la probada efectividad del amitraz (Semkiw et al., 2013), la poca disponibilidad de acaricidas específicos para las abejas en México y la necesidad del experimento de garantizar la reducción significativa de los niveles de infestación, independientemente de las condiciones ambientales de cada época del año.

Tamaño poblacional, reservas de alimento y peso. La población de abejas, áreas de cría, miel y polen de las colonias se calcularon a través del porcentaje promedio estimado por dos personas de la superficie de cada lado del panal ocupada por dichas variables. Para determinar la población de abejas, se utilizó la superficie porcentual y el número de abejas que ocupan un panal Langstroth de cámara de cría por ambos lados (2,430 abejas) (Delaplane et al., 2013). La superficie porcentual de cría, miel y polen se convirtió en área $\left(\mathrm{cm}^{2}\right)$, usando la superficie que tiene un panal tipo Langstroth en ambos lados $\left(1,760 \mathrm{~cm}^{2}\right)$ (Delaplane et al., 2013). Las mediciones se realizaron durante el periodo de la tarde (16 a $19 \mathrm{~h}$ ) cuando la mayoría de las abejas se encontraban en el interior de las colmenas. El peso de las colonias se determinó restando el peso del equipo (piso, cámara de cría, techo y panales) del peso total de cada colmena.

Las evaluaciones se realizaron con una frecuencia mensual a partir de mayo de 2016 a abril de 2017. Se suministró $1.5 \mathrm{~L}$ de jarabe de azúcar (1:1) semanalmente y $250 \mathrm{~g}$ de sustituto proteico $(25 \%$ proteína) cada dos semanas, cuando las condiciones ambientales no proveían alimento a las colonias (14 de junio al 22 de julio y del 10 de diciembre de 2016 al 20 de enero de 2017).

Infestación por $\boldsymbol{V}$. destructor. Se determinó el nivel de infestación en abejas adultas, en cría de obreras y el número de ácaros caídos en las colonias experimentales, cada mes a partir de mayo de 2016 a abril de 2017. El nivel de infestación en abejas se determinó mediante el método de De Jong al dividir el número de ácaros entre el total de abejas analizadas y multiplicado por 100 (De Jong et al., 1982). Se estimó el número total de ácaros en las abejas adultas de las colonias con el número promedio de ácaros por abeja multiplicado por la población de abejas estimada en las colonias (Delaplane et al., 2013). El nivel de infestación en la cría de obreras se determinó al dividir el número de celdas infestadas de una porción $(10 \times 10 \mathrm{~cm})$ de panal con cría operculada entre el número de celdas analizadas y multiplicando por 100 (De Jong et al., 1982).

Para el registro de ácaros caídos, se instaló en el piso de las colmenas una lámina galvanizada $(28 \times 43.5 \mathrm{~cm})$ impregnada con petrolato y entre la lámina y la cámara de cría se colocó una malla ( $3 \mathrm{~mm}$ ), esto para que los ácaros caídos pasen por la malla y se adhirieran a la lámina. El promedio diario de varroas caídas se obtuvo al dividir el número de ácaros registrados entre siete días que permanecieron colocadas las 
láminas adheribles (Dietemann et al., 2013). Además, durante la aplicación del tratamiento acaricida se registró semanalmente durante cuatro semanas la caída de ácaros en las láminas de los cuatro grupos de colonias.

Análisis estadístico. A partir de los registros mensuales, se determinó el promedio de cada una de las variables medidas (población de abejas, áreas $\left(\mathrm{cm}^{2}\right)$ de cría, miel y polen, peso, niveles de infestación en abejas adultas, en cría de obreras y el número de ácaros caídos) para cada estación del año, y se utilizaron pruebas de análisis de varianza, de medidas repetidas y la prueba de comparación de medias de NewmanKeuls. Además de pruebas de correlación de Pearson para establecer relaciones entre las variables evaluadas y la prueba de $\chi^{2}$ para determinar posibles diferencias en la frecuencia de casos de mortalidad de colonias entre las colonias tratadas con amitraz en verano, verano e invierno, invierno y testigo. Los datos porcentuales fueron transformados a raíz cuadrada del arcoseno, esto para normalizar su distribución (SAS, 2014).

\section{RESULTADOS}

Al inicio del experimento (abril de 2016), las 48 colonias seleccionadas contaron con condiciones estadísticamente similares de población de abejas ( $\mathrm{F}=0.14, \mathrm{P}=0.35)$, cría operculada $(F=0.42, P=0.52)$, reservas de miel $(F=0.14, P=0.78)$, polen $(F=0.39$, $\mathrm{P}=0.65)$ y niveles de infestación de $V$. destructor $(\mathrm{F}=0.44, \mathrm{P}=0.51)$.

Se observó reducción significativa en la población de varroas a consecuencia de los tratamientos acaricidas aplicados en verano e invierno. Los valores de los niveles de infestación en abejas adultas, cría y la caída diaria de varroas antes y después de los tratamientos aplicados en verano e invierno se muestran en los cuadros 1 y 2.

Al utilizar los registros mensuales de los niveles de infestación por Varroa y generar un promedio para cada estación del año, se observó que estos difieren significativamente entre los cuatro grupos de colonias $(\mathrm{P}<0.05)$, el tratamiento aplicado en el verano redujo los niveles de infestación en abejas adultas, cría y la caída de ácaros al fondo adherible de las colmenas. Sin embargo, en abejas adultas, los niveles de infestación se incrementaron rápidamente para el otoño a niveles similares a los cuales fueron tratadas inicialmente (figura 1).

La población de Varroa en la cría y los ácaros caídos en el piso adherible de las colmenas tuvieron un comportamiento similar (figuras 2 y 3 ), en ambos sitios, las colonias tratadas en verano (verano y verano e invierno) presentaron niveles significativamente $(F=7.01, P=0.0008)$ inferiores en las estaciones de verano y el otoño a diferencia de las colonias tratadas en el invierno y no tratadas. El tratamiento acaricida aplicado en el invierno solo tuvo efecto en la población de ácaros en abejas 
adultas. El número de ácaros en el fondo adherible y en la cría durante el invierno y la primavera, no difirieron entre los cuatro grupos de colonias (figuras 1, 2 y 3 ).

Cuadro 1. Nivel de infestación por $V$. destructor en abejas y cría (\% mediatee) y caída diaria (media $\pm e e)$ de varroas antes y después del tratamiento con amitraz en el verano, de colonias de los grupos experimentales: verano, invierno, verano e invierno y testigo.

\begin{tabular}{lccccc}
\hline \multicolumn{1}{c}{$\begin{array}{c}\text { Variable } / \text { grupo de } \\
\text { colonias }\end{array}$} & Verano & Invierno & $\begin{array}{c}\text { Verano e } \\
\text { invierno }\end{array}$ & Testigo & F y P \\
\hline $\begin{array}{l}\text { Infestación de abejas adultas } \\
\text { antes del tratamiento }\end{array}$ & $7.01 \pm 0.66 \mathrm{a}$ & $10.51 \pm 2.4 \mathrm{a}$ & $10.95 \pm 1.33 \mathrm{a}$ & $10.36 \pm 1.69 \mathrm{a}$ & $1.42,0.25$ \\
$\begin{array}{l}\text { Infestación de abejas adultas } \\
\text { después del tratamiento }\end{array}$ & $0.69 \pm 0.44 \mathrm{~b}$ & $13.98 \pm 2.7 \mathrm{a}$ & $0.94 \pm 0.50 \mathrm{~b}$ & $11.05 \pm 1.83 \mathrm{a}$ & $18.97,<0.0001$ \\
$\begin{array}{l}\text { Infestación en cría antes del } \\
\text { tratamiento }\end{array}$ & $11.0 \pm 1.75 \mathrm{a}$ & $8.40 \pm 1.84 \mathrm{a}$ & $10.09 \pm 2.23 \mathrm{a}$ & $10.82 \pm 1.54 \mathrm{a}$ & $0.39,0.76$ \\
$\begin{array}{l}\text { Infestación en cría después } \\
\text { del tratamiento }\end{array}$ & $2.31 \pm 0.57 \mathrm{~b}$ & $25.25 \pm 4.73 \mathrm{a}$ & $5.25 \pm 1.45 \mathrm{~b}$ & $19.34 \pm 2.58 \mathrm{a}$ & $17.68,<0.0001$ \\
$\begin{array}{l}\text { Caída diaria de varroas antes } \\
\text { del tratamiento }\end{array}$ & $51.13 \pm 9.2 \mathrm{a}$ & $77.0 \pm 15 \mathrm{a}$ & $72.7 \pm 10 \mathrm{a}$ & $63.9 \pm 11 \mathrm{a}$ & $1.01,0.39$ \\
$\begin{array}{l}\text { Caída diaria de varroas } \\
\text { después del tratamiento }\end{array}$ & $38.2 \pm 3.7 \mathrm{~b}$ & $66.7 \pm 14.8 \mathrm{ab}$ & $36.9 \pm 4 \mathrm{~b}$ & $113.58 \pm 31 \mathrm{a}$ & $4.46,0.008$ \\
\hline $\begin{array}{l}\text { Valores sin transformar. Diferentes literales entre filas indican diferencias significativas basadas en un } \\
\text { análisis de varianza y la comparación de medias con la prueba de Newman-Keuls, previa transformación } \\
\text { de datos. }\end{array}$ & & & &
\end{tabular}

Cuadro 2. Nivel de infestación por $V$. destructor en abejas y cría (\% media $\pm e e)$ y caída diaria (mediatee) de varroas antes y después del tratamiento con amitraz en el invierno, de colonias de los grupos experimentales: verano, invierno, verano e invierno y testigo.

\begin{tabular}{|c|c|c|c|c|c|}
\hline Variable & Verano & Invierno & $\begin{array}{l}\text { Verano e } \\
\text { invierno }\end{array}$ & Testigo & F y $P$ \\
\hline $\begin{array}{l}\text { Infestación de abejas } \\
\text { adultas antes del tratamiento } \\
\text { Infestación de abejas }\end{array}$ & $10.77 \pm 1.07 a$ & $10.43 \pm 1.69 a$ & $9.41 \pm 1.48 a$ & $9.73 \pm 1.42 a$ & $0.22,0.88$ \\
\hline $\begin{array}{l}\text { adultas después del } \\
\text { tratamiento }\end{array}$ & $5.68 \pm 0.72 a$ & $0.09 \pm 0.04 b$ & $0.47 \pm 0.33 b$ & $7.2 \pm 1.22 \mathrm{a}$ & $18.97,<0.0001$ \\
\hline $\begin{array}{l}\text { Infestación en cría antes del } \\
\text { tratamiento }\end{array}$ & $7.88 \pm 1.25 \mathrm{a}$ & $16.22 \pm 2.24 a$ & $10.4 \pm 2.2 a$ & $14.1 \pm 3.63 a$ & $2.55,0.070$ \\
\hline $\begin{array}{l}\text { Infestación en cría después } \\
\text { del tratamiento }\end{array}$ & $0.62 \pm 0.24 b$ & $0.45 \pm 0.30 \mathrm{~b}$ & $0.20 \pm 0.10 b$ & $2.20 \pm 0.85 a$ & $2.89,0.045$ \\
\hline $\begin{array}{l}\text { Caída diaria de varroas } \\
\text { antes del tratamiento }\end{array}$ & $20.7 \pm 4.3 a$ & $16.8 \pm 4.2 \mathrm{a}$ & $21.3 \pm 3.6 a$ & $17.6 \pm 3.5 \mathrm{a}$ & $0.29,0.82$ \\
\hline $\begin{array}{l}\text { Caída diaria de varroas } \\
\text { después del tratamiento }\end{array}$ & $1.75 \pm 0.48 b$ & $1.31 \pm 0.30 \mathrm{~b}$ & $1.36 \pm 0.25 b$ & $3.8 \pm 0.9 a$ & $4.35,0.01$ \\
\hline
\end{tabular}




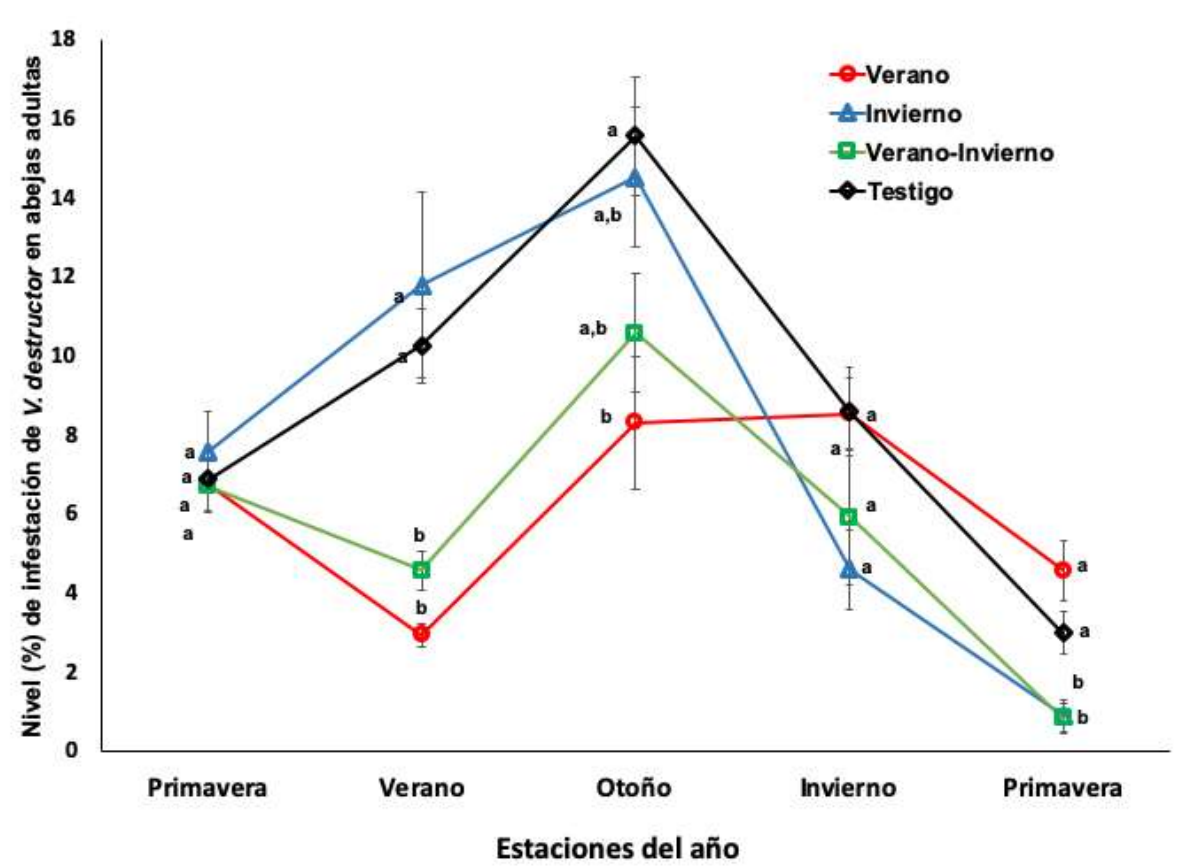

Figura 1. Nivel de infestación (media \pm ee) de $V$. destructor en abejas adultas de colonias tratadas con amitraz en verano, invierno, verano e invierno y no tratadas. Valores sin transformar. Diferentes literales indican diferencias significativas $(P<0.05)$ basadas en un análisis de mediciones repetidas y la comparación de medias con la prueba de Newman-Keuls, previa transformación de datos al arcoseno de la raíz cuadrada.

Respecto a la caída de varroas en el piso adherible de las colmenas durante las cuatro aplicaciones del tratamiento acaricida, se observó que la primera aplicación del tratamiento provocó significativamente $(\mathrm{F}=8.51, \mathrm{P}<0.001)$ mayor número de ácaros caídos que en las aplicaciones posteriores. La caída de ácaros debida a las últimas tres aplicaciones del amitraz fueron estadísticamente similares $(P>0.05)$ a la caída natural del ácaro del grupo testigo.

La población de abejas, áreas de miel, polen y peso no fueron diferentes entre los cuatro grupos de colonias en las cuatro estaciones del año. Solo se observaron diferencias significativas en las áreas de cría operculada durante el otoño, las colonias tratadas en verano $\left(6,747 \pm 352 \mathrm{~cm}^{2}\right)$ y verano-invierno $\left(5,960 \pm 191 \mathrm{~cm}^{2}\right)$ tuvieron significativamente $(F=3.55, P=0.023)$, mayores áreas de cría que las colonias tratadas en el invierno $\left(4,889 \pm 472 \mathrm{~cm}^{2}\right)$ o no tratadas $\left(5,240 \pm 631 \mathrm{~cm}^{2}\right)$, entre las cuales no hubo diferencia significativa.

Sin considerar el tratamiento al cual pertenecían las colonias, las áreas de cría operculada y la población de abejas fueron estadísticamente inferiores en las últimas dos estaciones del experimento (invierno y primavera) a diferencia de lo observado en las primeras estaciones, dichos valores se presentan en el cuadro 3. 


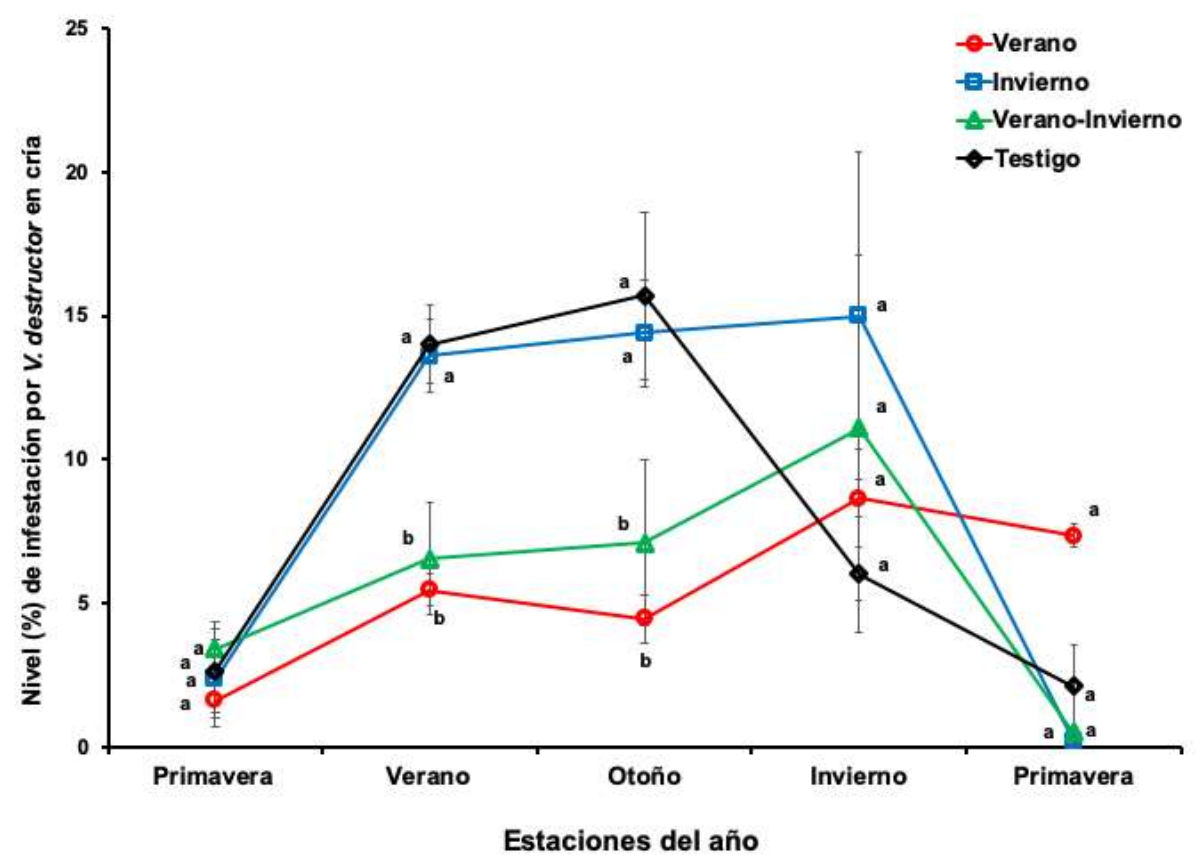

Figura 2. Nivel de infestación (mediatee) de $V$. destructor en cría de colonias tratadas con amitraz en verano, invierno, verano e invierno y no tratadas. Valores sin transformar. Diferentes literales indican diferencias significativas $(\mathrm{P}<0.05)$ basadas en un análisis de mediciones repetidas con valores transformados y la comparación de medias con la prueba de Newman-Keuls.

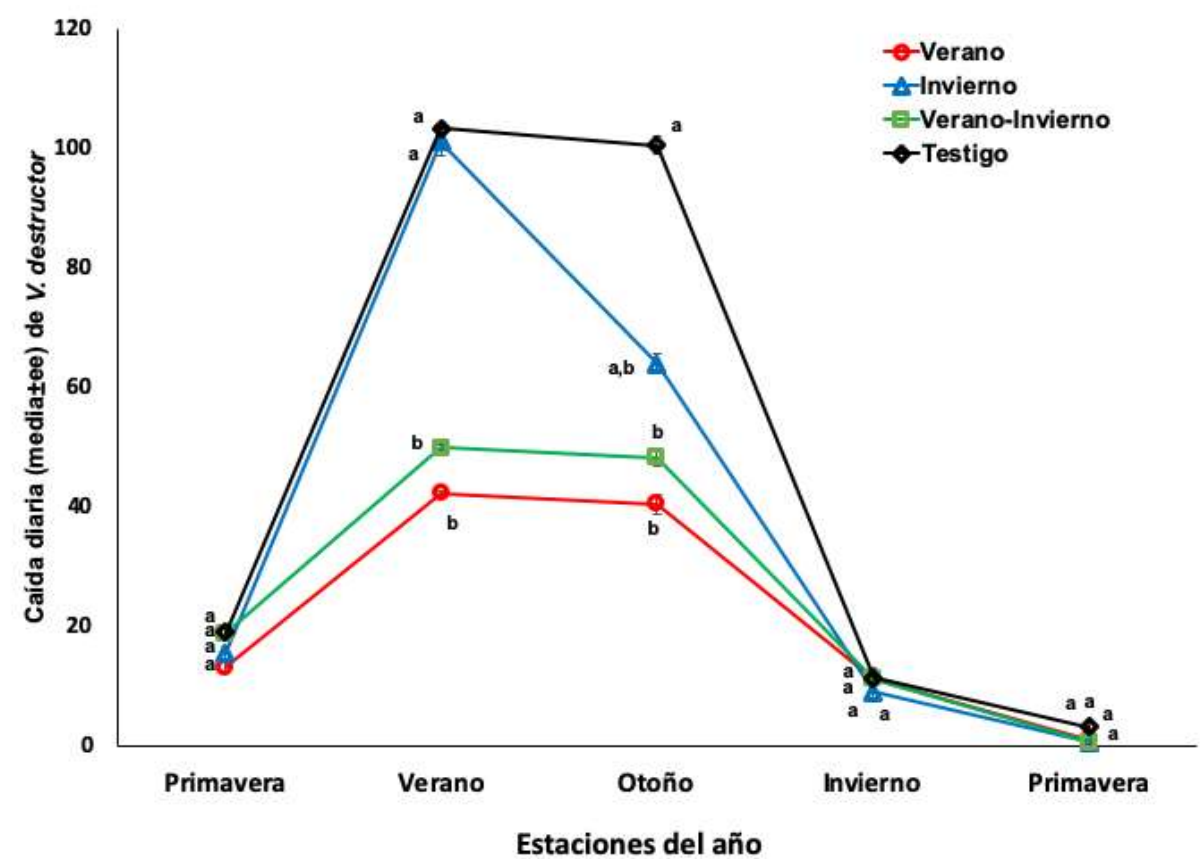

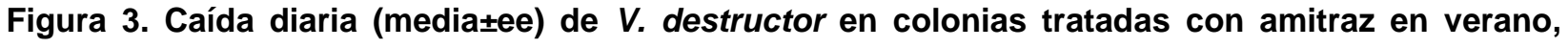
invierno, verano e invierno y no tratadas. Diferentes literales indican diferencias significativas basadas en un análisis de mediciones repetidas y la comparación de medias con la prueba de Newman-Keuls $(\mathrm{P}<0.05)$. 
Cuadro 3. Áreas $\left(\mathrm{cm}^{2}\right)$ de cría operculada y población de abejas adultas de colonias de abejas melíferas $(n=48)$ durante la primavera, verano, otoño e invierno de 2016 y la primavera de 2017.

\begin{tabular}{lll}
\hline Estación & Áreas de cría $\left(\mathbf{c m}^{2}\right)$ & Población de abejas \\
\hline Primavera (2016) & $10,410 \pm 182 \mathrm{a}$ & $9,484 \pm 136 \mathrm{a}, \mathrm{b}$ \\
Verano (2016) & $8,387 \pm 220 \mathrm{~b}$ & $10,011 \pm 398 \mathrm{a}$ \\
Otoño (2016) & $5,771 \pm 238 \mathrm{c}$ & $8,614 \pm 417 \mathrm{~b}$ \\
Invierno (2016) & $3,252 \pm 240 \mathrm{~d}$ & $4,366 \pm 372 \mathrm{c}$ \\
Primavera (2017) & $5,813 \pm 275 \mathrm{c}$ & $5,123 \pm 445 \mathrm{c}$ \\
F y P & $\mathrm{F}=146.8, \mathrm{P}<0.0001$ & $\mathrm{~F}=48.9, \mathrm{P}<0.0001$ \\
\hline
\end{tabular}

Diferentes literales en cada columna indican diferencias significativas basadas en un análisis de varianza y la comparación de medias con la prueba de Newman-Keuls.

Al final del experimento (primavera), el número promedio de varroas por abeja y el tamaño poblacional de varroas estimado en la población de abejas adultas de las colonias fue significativamente menor en los grupos tratados en el invierno (invierno y verano e invierno), pero no se presentaron diferencias significativas respecto a los valores poblacionales, reservas de alimento y peso entre las colonias tratadas en verano, invierno, verano e invierno y el grupo testigo (cuadro 4).

Cuadro 4. Población de abejas, áreas $\left(\mathrm{cm}^{2}\right)$ de cría, miel, polen, peso, promedio de varroas por abeja y población de ácaros en abejas adultas de colonias tratadas con amitraz en el verano, invierno, verano e invierno y no tratadas, al término del experimento (primavera).

\begin{tabular}{cccccccc}
\hline Grupo & $\begin{array}{c}\text { Población } \\
\text { de abejas }\end{array}$ & $\begin{array}{c}\text { Población } \\
\text { de cría } \\
\left(\mathbf{c m}^{2}\right)\end{array}$ & $\begin{array}{c}\text { Reservas } \\
\text { de miel } \\
\left.\mathbf{( c m}^{2}\right)\end{array}$ & $\begin{array}{c}\text { Reservas } \\
\text { de polen } \\
\left.\mathbf{( c m}^{2}\right)\end{array}$ & $\begin{array}{c}\text { Peso } \\
\mathbf{( K g )}\end{array}$ & $\begin{array}{c}\text { Varroas por } \\
\text { cada } \mathbf{1 0 0} \\
\text { abejas }\end{array}$ & $\begin{array}{c}\text { Ácaros en } \\
\text { población } \\
\text { de abejas } \\
\text { estimada }\end{array}$ \\
\hline Verano & $13314 \pm 953$ & $5560 \pm 475$ & $3560 \pm 372$ & $660 \pm 220$ & $8.5 \pm 1.02$ & $0.045 \pm 0.0 \mathrm{a}$ & $602 \pm 114 \mathrm{a}$ \\
Invierno & $12757 \pm 1349$ & $6356 \pm 464$ & $3276 \pm 544$ & $605 \pm 321$ & $6.2 \pm 1.03$ & $0.0086 \pm 0.01 \mathrm{~b}$ & $109 \pm 50 \mathrm{~b}$ \\
Verano- & $12879 \pm 1542$ & $5808 \pm 593$ & $3036 \pm 266$ & $968 \pm 205$ & $7.3 \pm 0.81$ & $0.0078 \pm 0.00 \mathrm{~b}$ & $100 \pm 42 \mathrm{~b}$ \\
Invierno & & & & & & & \\
Testigo & $14200 \pm 1847$ & $5555 \pm 743$ & $3410 \pm 360$ & $1320 \pm 525$ & $6.7 \pm 0.85$ & $0.0293 \pm 0.01 \mathrm{a}$ & $416 \pm 86 \mathrm{a}$ \\
& $\mathrm{F}=0.19$, & $\mathrm{F}=0.43$, & $\mathrm{F}=0.34$, & $\mathrm{F}=1.01$, & $\mathrm{F}=1.13$, & $\mathrm{F}=10.09$, & $\mathrm{F}=8.71$, \\
& $\mathrm{P}=0.90$ & $\mathrm{P}=0.73$ & $\mathrm{P}=0.79$ & $\mathrm{P}=0.39$ & $\mathrm{P}=0.35$ & $\mathrm{P}<0.0001$ & $\mathrm{P}=0.0002$ \\
\hline
\end{tabular}

Diferentes literales en cada columna indican diferencias significativas basadas en un análisis de varianza y la comparación de medias con la prueba de Newman-Keuls.

El nivel de infestación en abejas se relacionó positiva y significativamente con el nivel de infestación en cría $(r=0.52, P=0.0002)$ y con la caída de ácaros al piso adherible de las colmenas $(r=0.63, P<0.0001)$. Asimismo, el peso de las colonias se relacionó con la población de abejas de las colonias $(r=0.65, P<0.001)$. 
En el grupo de colonias tratadas en el verano murió el $8 \%$ de las colonias y el $16 \%$ en el grupo tratado en el verano e invierno, mientras que en el grupo tratado en el invierno y testigo hubo una mortalidad del $25 \%$ y $33 \%$, respectivamente, no hubo diferencias estadísticas significativas $\left(X^{2}=2.52, \mathrm{gl}=3, \mathrm{P}=0.47\right)$.

\section{DISCUSIÓN}

La reducción en la población de varroas a consecuencia de los tratamientos aplicados en verano e invierno confirman la efectividad acaricida del amitraz al disminuir significativamente la población de ácaros en las colonias de abejas (Semkiw et al., 2013). Sin embargo, se observó un rápido incremento de los niveles de infestación en abejas adultas en el otoño en las colonias tratadas durante el verano, lo cual se ha presentado en otras investigaciones y ha sido atribuido a varroas sobrevivientes al tratamiento y a la migración de ácaros procedentes de colonias no tratadas del mismo apiario (Delaplane y Hood, 1997; Gatien y Currie, 2003; Wilfert et al., 2016).

Considerando los promedios de infestación para cada estación del año, se observó que el tratamiento acaricida aplicado en el invierno solo tuvo efecto en la población de ácaros en abejas adultas ( $\approx 7.3 \%$ menos respecto a los niveles de infestación en otoño) y que el número de ácaros en el fondo adherible en las colmenas (media=10.5) y en la cría (media $=10.1 \%$ ) durante el invierno y la primavera (1.4 y $2.5 \%$, respectivamente), no difirieron $(P>0.05)$ entre los cuatro grupos de colonias. En los escasos estudios con los que se puedan comparar los resultados del presente, se observa variabilidad en los resultados y algunos coinciden a los obtenidos en el presente estudio, tal es el caso del experimento realizado por Delaplane y Hood (1997) quienes no encontraron una reducción en el número de ácaros en las colonias tratadas en junio (1,702 varroas) en comparación con las colonias no tratadas (986), esto en el estado de Georgia EUA. Sin embargo, en dicho estudio encontraron que para Carolina del Sur el efecto del tratamiento sobre la población de ácaros fue significativo.

La dinámica poblacional de $V$. destructor se relaciona con el ciclo y la cantidad de cría disponible en la colonia (Delaplane, 1998; Caron, 1999). Y el incremento de la población de abejas y cría reduce la proporción de ácaros respecto a la población de abejas (Moretto et al., 1991). Lo anterior explica la notable reducción de los niveles de infestación al final del experimento en los cuatro grupos de colonias, ya que se presentó una reducción significativa de las áreas de cría y población de abejas en otoño e invierno ( $\mathrm{P}<0.0001$, cuadro 3 ), lo que ocasionó menores posibilidades de reproducción para el ácaro. Adicionalmente, en la primavera, el incremento observado en las áreas de cría y población de abejas derivado de una mayor disponibilidad de recursos para las abejas pudo reducir la proporción de varroas respecto a la población de abejas. 
En relación al número de aplicaciones del tratamiento, los resultados revelan que en la primera dosis hubo mayor $(\mathrm{P}<0.001)$ caída de ácaros respecto a las posteriores aplicaciones y al grupo testigo. Lo anterior probablemente se deba a que durante la primera aplicación muere la mayoría de los ácaros y el reducido número de varroas remanentes en fase forética y reproductiva no permita identificar diferencias significativas respecto al grupo testigo en las posteriores aplicaciones del tratamiento. Estos resultados coinciden con los reportados previamente con el uso de timol (Espinosa-Montaño y Guzmán-Novoa, 2007). No obstante, es importante mantener el tratamiento durante la emergencia de nuevas varroas que se encuentran en etapa reproductiva al interior de las celdillas, esto permitirá lograr una mejor reducción de la población de ácaros en las colonias (Rosenkranz et al., 2010).

La población de abejas, áreas de miel, polen y peso no fueron diferentes entre los cuatro grupos de colonias en las cuatro estaciones del año. Solo se observaron diferencias significativas en las áreas de cría operculada durante el otoño, las colonias tratadas en verano $\left(6,747 \pm 352 \mathrm{~cm}^{2}\right)$ y verano e invierno $\left(5,960 \pm 191 \mathrm{~cm}^{2}\right)$ tuvieron significativamente $(P=0.023)$, mayores áreas de cría que las colonias tratadas en el invierno $\left(4,889 \pm 472 \mathrm{~cm}^{2}\right)$ o no tratadas $\left(5,240 \pm 631 \mathrm{~cm}^{2}\right)$, entre las cuales no hubo diferencia significativa.

A excepción de los tratamientos aplicados en el verano, los cuales repercutieron con mayores áreas de cría en el otoño $(P=0.023)$; Las diferencias en los niveles de infestación de Varroa entre los tratamientos (colonias tratadas en verano, invierno, verano-invierno y testigo) no tuvieron efectos significativos en las variables de población (abejas y áreas de cría), reservas de alimentos (miel y polen) y peso de las colonias. Al igual que en el presente estudio, en los trabajos realizados por Delaplane y Hood $(1997,1999)$ y Strange y Sheppard (2001) se muestran resultados contradictorios sobre el efecto de los niveles de infestación de $V$. destructor sobre los parámetros poblacionales de las colonias, sus reservas alimenticias y el peso de las colonias. Al respecto, se ha reportado que no siempre existe una clara relación entre la población de ácaros y la población de la colonia (Korpela et al., 1992) y que los tratamientos acaricidas en ocasiones no repercuten en la producción de crías en colonias parasitadas por Varroa (Delaplane, 1995). Probablemente esto se deba a una mayor producción de cría en colonias con altos niveles de infestación por Varroa debido al esfuerzo de las colonias para compensar la pérdida de crías a consecuencia de la parasitosis (Delaplane y Hood, 1997).

Al final del experimento (primavera), los niveles de infestación promedio de 0.045 ácaros por abeja (4.5\% de infestación) y una población estimada de 100 a 602 ácaros, no redujeron la población de abejas, áreas de cría, miel, polen y peso de las colonias del altiplano central de México con poblaciones de 12,757 a 14,200 abejas. En este sentido, se ha reportado que el nivel de infestación tolerable para la colonia cambia 
regionalmente debido a la variación en el período de cría en las colonias y sus efectos sobre la dinámica poblacional del ácaro (Delaplane, 1998; Caron, 1999).

En México, la SAGARPA (2005) recomienda que a partir del 5\% de infestación por Varroa en abejas y/o 10 ácaros caídos en $24 \mathrm{~h}$ al piso adherible de las colmenas se implemente algún tratamiento contra la enfermedad. Sin embargo, estos umbrales difieren a otros establecidos por diferentes autores en diferentes épocas y regiones. El umbral de tratamiento establecido para el noroeste y suroeste de EU es $5 \%$ en abejas y de 12 ácaros caídos en 24 h en primavera (Strange y Sheppard, 2001). Para el verano se recomienda que el tratamiento acaricida se aplique a partir de 23 ácaros para el noroeste y 70 a 224 caídos en 24 h o de 3,000 a 4,000 ácaros para colonias con poblaciones de 24,000 a 34,000 abejas (8-8.5\% en abejas) en el sureste de los EU (Delaplane y Hood, 1997,1999). En contraste, para Reino Unido el umbral de daño ha sido reportado de 2,500 ácaros por colonia (Martin, 1999).

En Canadá, los niveles promedio de infestación por Varroa de $2 \%$ en abejas tienen un efecto negativo sobre la producción de miel y en colonias con niveles superiores al $4 \%$ en verano es necesario aplicar un tratamiento para prevenir su pérdida en otoño e invierno (Currie y Gatien, 2006). Contradictoriamente, Currie y Gatien (2006) reportan que niveles de 7 y $10 \%$ en abejas no repercuten sobre la producción de miel de las colonias. En Valle de Bravo, estado de México, se observó que colonias tratadas con fluvalinato y con un promedio de infestación del $2.3 \%$ produjeron $65 \%$ más miel que colonias no tratadas y con un nivel promedio de infestación del 6.8\% (Arechavaleta y Guzmán-Novoa, 2000). Lo anterior refleja variabilidad de efectos que los niveles de infestación ejercen sobre las colonias de abejas melíferas en diferentes condiciones ambientales y épocas del año. Probablemente, la presencia de virus y otros factores sanitarios podrían explicar las variaciones encontradas en los umbrales de tratamiento reportados.

El análisis de correlación permitió observar una relación positiva entre los niveles de infestación en abejas y cría y con la caída diaria de ácaros, así como el peso con la población de abejas de las colonias. Lo anterior coincide con lo reportado por Gąbka (2014) y Saini (2018). Estas características pueden ser utilizadas por los apicultores como estrategias predictivas de las condiciones de las colonias.

Se ha reportado que colonias que reciben un tratamiento acaricida a inicios del año presentan una menor tasa de mortalidad a diferencia de colonias tratadas tardíamente 0 no tratadas (Delaplane y Hood, 1997; Fries et al., 2006). Sin embargo, en el presente estudio la tasa de mortalidad de los cuatro grupos de colonias no difirió significativamente. 
Se requieren estudios adicionales que proporcionen mayor información respecto al efecto del tratamiento contra Varroa aplicado en diferentes estaciones del año sobre los niveles de infestación del ácaro y las condiciones poblacionales y alimenticias, así como su efecto en la producción de colonias de abejas melíferas en diferentes regiones.

\section{CONCLUSIÓN}

El tratamiento aplicado en el verano redujo significativamente los niveles de infestación por Varroa, pero estos se incrementaron para el otoño a niveles similares a los iniciales. Las diferencias en los niveles de infestación observadas en otoño, invierno y primavera no repercutieron en la población de abejas, áreas de cría, miel, polen y peso de las colonias. Niveles de infestación promedio de $4.5 \%$ en abejas y una población estimada de hasta 602 ácaros, no redujeron la población, reservas de alimento y peso de las colonias evaluadas en el presente estudio con poblaciones de hasta 14,200 abejas. Se recomienda monitorear el nivel de infestación de las colonias y aplicar acaricidas durante el invierno para evitar que la población de ácaros se incremente y repercuta sobre las condiciones y productividad de las colonias de abejas.

\section{AGRADECIMIENTOS}

Se agradece a la Unidad Académica de Medicina Veterinaria y Zootecnia de la Universidad Autónoma de Zacatecas, al Colegio de Posgraduados Campus San Luis Potosí por las facilidades otorgadas para la realización del presente estudio y la obtención del grado de Maestro en Ciencias en Innovación de Manejo de Recursos Naturales del primer autor, así como al CONACYT por la beca otorgada.

\section{LITERATURA CITADA}

ANDERSON DL, Trueman JWH. 2000. Varroa jacobsoni (Acari: Varroidae) is more than one species. Experimental and Applied Acarology. 24(3):165-189. ISSN: 1572-9702. https://doi.org/10.1023/A:1006456720416

ARECHAVALETA VME, Guzmán-Novoa E. 2000. Producción de miel en colonias de abejas (Apis mellifera $L$.) tratadas y no tratadas con fluvalinato contra Varroa jacobsoni Oudemans en Valle de bravo, Estado de México. Veterinaria México. 31(4):381384.ISSN 2448-6760. https://www.medigraphic.com/pdfs/vetmex/vm-2000/vm004m.pdf

BERRY JA, Hood WM, Pietravalle S, Delaplane KS. 2013. Field-Level Sublethal Effects of Approved Bee Hive Chemicals on Honey Bees (Apis mellifera L). PLoS ONE. 8(10): e76536. ISSN: 1932-6203. https://doi.org/10.1371/journal.pone.0076536

BONCRISTIANI H, Underwood R, Schwarz R, Evans JD, Pettis J, vanEngelsdorp D. 2012. Direct effect of acaricides on pathogen loads and gene expression levels in honey bees Apis mellifera. Journal of Insect Physiology. 58:613-620. ISSN: 00221910. https://doi.org/10.1016/j.jinsphys.2011.12.011 
CARON D. 1999. Delaware bee mites survey. American Bee Journal. 139(8): 631-633. ISSN: 00027626.

https://europepmc.org/article/agr/ind22002953?client=bot\&client=bot\&client=bot

CURRIE RW, Gatien P. 2006. Timing acaricide treatments to prevent Varroa destructor (Acari: Varroidae) from causing economic damage to honey bee colonies. The Canadian Entomologist. 138(2):238-252. ISSN: 0008-347X. https://doi.org/10.4039/n05-024

DAINAT B, Evans JD, Chen YP, Gauthier L, Neumann P. 2012. Dead or alive: deformed wing virus and Varroa destructor reduce the life span of winter honeybees. Applied Environmental Microbiology. 78(4):981-987. ISSN: 1098-5336. http://dx.doi.org/10.1128/AEM.06537-11

DELAPLANE KS, Der-Steen JV, Guzman-Novoa E. 2013. Standard methods for estimating strength parameters of Apis mellifera colonies. Journal of Apicultural Research. 52(1):1-12. ISSN: 0021-8839. https://doi.org/10.3896/IBRA.1.52.1.03

DELAPLANE KS, Hood WM. 1997. Effects of delayed acaricide treatment in honey bee colonies parasitized by Varroa jacobsoni and a late-season treatment threshold for the south-eastern USA. Journal of Apicultural Research. 36(3/4):125-132. ISSN: 0021-8839. https://doi.org/10.1080/00218839.1997.11100938

DELAPLANE KS, Hood WM. 1999. Economic threshold for Varroa jacobsoni Oud. in the southeastern USA. Apidologie. 30: 383-395. ISSN: 0044-8435. https://doi.org/10.1051/apido:19990504

DELAPLANE KS. 1995. Effects of Terramycin antibiotic and Apistan acaricide on colonies of honey bees (Hymenoptera: Apidae) infested with Varroa jacobsoni (Parasitiformes: Varroidae). Journal of Economic Entomology. 88(5):1206-1210. ISSN 0022-0493. https://doi.org/10.1093/jee/88.5.1206

DELAPLANE KS. 1998. Varroa control: timing is everything. American Bee Journal. 138:575-576. ISSN: 0002-7626. $\quad$ https://agris.fao.org/agrissearch/search.do?recordlD=US1997076691

DIETEMANN V, Nazzi F, Martin SJ, Anderson D, Locke B, Delaplane K, Wauquiez Q, Tannahill C, Frey E, Ziegelmann B, Rosenkranz P, Ellis JD. 2013. Standard methods for varroa research. Journal of Apicultural Research. 52(1):1-54. ISSN: 0021-8839. https://doi.org/10.3896/IBRA.1.52.1.09

DE JONG D, Roma DA, Goncalves LS. 1982. A comparative analysis of shaking solutions for the detection of Varroa jacobsoni on adult honeybees. Apidologie. 13:297306. ISSN: 1297-9678.

https://www.apidologie.org/articles/apido/pdf/1982/03/Apidologie_0044-

8435_1982_13_3_ART0008.pdf 
ESPINOSA-MONTAÑO LG, Guzmán-Novoa E. 2007. Eficacia de dos acaricidas naturales, ácido fórmico y timol, para el control del ácaro Varroa destructor de las abejas (Apis mellifera L.) en Villa Guerrero, Estado de México. Veterinaria México 38(1):9-19. ISSN: 0301-5092. http://www.redalyc.org/articulo.oa?id=42338102

FRIES I, Imdorf A, Rosenkranz P. 2006. Survival of mite infested (Varroa destructor) honey bee (Apis mellifera) colonies in a Nordic climate. Apidologie. 37:564-570. ISSN: 0044-8435. https://doi.org/10.1051/apido:2006031

GĄBKA J. 2014. Correlations between the strength, amount of brood and honey production of the honey bee colony. Medycyna Weterynaryjna. 70(12):754-756. ISSN: 0025-8628.

https://www.researchgate.net/profile/Jakub_Gbka/publication/293074876_Correlations_ between_the_strength_amount_of_brood_and_honey_production_of_the_honey_bee_c olony/links/58385ea008ae3d91723dd7f7/Correlations-between-the-strength-amount-ofbrood-and-honey-production-of-the-honey-bee-colony.pdf

GASHOUT HA, Goodwin PH, Guzman-Novoa E. 2018. Lethality of synthetic and natural acaricides to worker honey bees (Apis mellifera) and their impact on the expression of health and detoxification-related genes. Environmental Science and Pollution Research. 25(34): 34730-34739. ISSN: 09441344. https://doi.org/10.1007/s11356-018-3205-6

GASHOUT HA, Guzman-Novoa E, Goodwin PH, Correa-Benítez A. 2020. Impact of sublethal exposure to synthetic and natural acaricides on honey bee (Apis mellifera) memory and expression of genes related to memory. Journal of Insect Physiology. 121 104014. ISSN: 00221910. https://doi.org/10.1016/j.jinsphys.2020.104014

GATIEN P, Currie RW. 2003. Timing of acaracide treatments for control of low-level populations of Varroa destructor (Acari: Varroidea) and implications for colony performance of honey bee. The Canadian Entomologist. 135(5):749-763. ISSN: 0008347X. https://doi.org/10.4039/n02-086

GONZÁLEZ-CABRERA J, Rodríguez-Vargas S, Davies TGE, Field LM, Schmehl D, Ellis JD, Krieger K, Williamson MS. 2016. Novel Mutations in the Voltage-Gated Sodium Channel of Pyrethroid-Resistant Varroa destructor Populations from the Southeastern USA. PLOS ONE. 11(5): e0155332. ISSN: 1932-6203.

https://doi.org/10.1371/journal.pone.0155332

GREGORC A, Planinc I. 2012. Use of thymol formulations, amitraz, and oxalic acid for the control of the varroa mite in honey bee (Apis mellifera carnica) colonies. Journal of Apicultural Science. 56(2): 61-69. ISSN: 2299-4831. https://doi.org/10.2478/v10289-0120024-8 
GUZMÁN-NOVOA E, Eccles L, Calvete Y, McGowan J, Kelly PG, Correa-Benítez A. 2010. Varroa destructor is the main culprit for the death and reduced populations of overwintered honey bee (Apis mellifera) colonies in Ontario, Canada. Apidologie. 41(4): 443-450. ISSN: 0044-8435. https://doi.org/10.1051/apido/2009076

INEGI. 2019. Prontuario de información geográfica municipal de los Estados Unidos Mexicanos. Instituto Nacional de Estadística Geografía e Informática. Consultado el 8 de junio de 2019 en: https://www.inegi.org.mx/app/areasgeograficas/?ag=32

KAMLER M, Nesvorna M, Stara J, Erban T, Hubert J. 2016. Comparison of taufluvalinate, acrinathrin, and amitraz effects on susceptible and resistant populations of Varroa destructor in a vial test. Experimental and applied acarology.69(1): 1-9. https://doi.org/10.1007/s10493-016-0023-8

KORPELA S, Aarhus A, Fries I, Hansen H. 1992. Varroa jacobsoni Oud. in cold climates: population growth, winter mortality and influence on the survival of honey bee colonies. Journal of Apicultural Research. 31(3/4): 157-164. ISSN: 0021-8839. https://doi.org/10.1080/00218839.1992.11101278

LUPO A, Gerling D. 1990. A comparison between the efficiency of summer treatments using formic acid and Taktic $®$ against Varroa jacobsoni in beehives. Apidologie. 21(3): 261-267. ISSN: 1297-9678.

https://doi.org/10.1051/apido:19900311

MARIE-PIERRE C, Anne-Claire M, Zeggane S, Drajnudel P, Schurr F, Marie-Claude C, Ribière-Chabert M, Aubent M, Jean-Paul F. 2010. A case control study and a survey on mortalities of honey bee colonies (Apis mellifera) in France during the winter of 2005-6. Journal of Apiculture Research. 49(1):40-51. ISSN: 0021-8839. https://doi.org/10.3896/IBRA.1.49.1.06

MARTIN SJ, Ball BV, Carreck NL. 2010. Prevalence and persistence of deformed wing virus (DWV) in untreated or acaricide-treated Varroa destructor infested honey bee (Apis mellifera) colonies. Journal of Apicultural Research. 49(1): 72-79. ISSN: 0021-8839. https://doi.org/10.3896/IBRA.1.49.1.10

MARTIN SJ. 1999. Population modeling and the production of a monitoring tool for Varroa jacobsoni an ectoparasitic mite of honey bees. Aspects of Applied Biology. 53: 105-112. ISSN: 0265-1491.

https://www.researchgate.net/publication/291772610_Population_modelling_and_the_pr oduction_of_a_monitoring_tool_for_Varroa_jacobsoni_an_ectoparasitic_mite_of_honey bees\#fullTextFileContent 
MARTÍNEZ-PUC JF, Medina-Medina LA. 2011. Evaluación de la resistencia del ácaro Varroa destructor al fluvalinato en colonias de abejas (Apis mellifera) en Yucatán, México. Revista Mexicana de Ciencias Pecuarias. 2(1):93-100. ISSN 2448-6698. https://cienciaspecuarias.inifap.gob.mx/index.php/Pecuarias/article/view/1451

MEDINA-FLORES CA, Guzmán-Novoa E, Aréchiga-Flores CF, Aguilera-Soto Jl, Gutiérrez-Piña J. 2011. Efecto del nivel de infestación de Varroa destructor sobre la producción de miel de colonias de Apis mellifera en el altiplano semiárido de México. Revista Mexicana de Ciencias Pecuarias. 2:313-317. ISSN 2448-6698. http://www.scielo.org.mx/scielo.php?script=sci_arttext\&pid=S2007-11242011000300006

MORETTO G, Goncalves SL, De Jong D. 1991. Africanized bees are more efficient at removing Varroa jacobsoni- Preliminary data. American Bee Journal. 131(7):434. ISSN: $0002-7626$.

https://pascal-francis.inist.fr/vibad/index.php?action=getRecordDetail\&idt=19855220

NAZZI F, Le Conte Y. 2016. Ecology of Varroa destructor, the major ectoparasite of the western honey bee, Apis mellifera. Annual Review of Entomology. 61:417-432. ISSN: 1545-4487. https://doi.org/10.1146/annurev-ento-010715-023731

PIETROPAOLI M, Formato G. 2018. Liquid formic acid $60 \%$ to control varroa mites (Varroa destructor) in honey bee colonies (Apis mellifera): protocol evaluation. Journal of Apicultural Research. 57(2): 300-307. ISSN: 0021-8839. https://doi.org/10.1080/00218839.2017.1376767

RODRÍGUEZ-DEHAIBES SR, Otero-Colina G, Villanueva-Jiménez JA, Corcuera P. 2011. Susceptibility of Varroa destructor (Gamasida: Varroidae) to four pesticides used in three mexican apicultural regions under two different management systems. International Journal of Acarology.37(5): 441-447. ISSN: 1572-9702. https://doi.org/10.1080/01647954.2010.525523

ROSENKRANZ P, Aumeier P, Ziegelmann B. 2010. Biology and control of Varroa destructor. Journal of invertebrate pathology. 103: S96-S119. ISSN: 1096-0805. https://doi.org/10.1016/j.jip.2009.07.016

RYABOV EV, Childers AK, Chen Y, Madella S, Nessa A, Evans JD. 2017. Recent spread of Varroa destructor virus-1, a honey bee pathogen, in the United States. Scientific reports. 7(1):1-10. ISSN: 2045-2322. https://doi.org/10.1038/s41598017-17802-3

SAGARPA (Secretaria de Agricultura, Ganadería, Desarrollo Rural, Pesca y Alimentación). 2005. Modificación a la Norma Oficial Mexicana NOM-001-ZOO-1994, campaña contra la varroasis de las abejas. Diario Oficial. 28 de diciembre de 2005. http://www.ordenjuridico.gob.mx/Federal/PE/APF/APC/SAGARPA/Modificaciones/28122 005(1).pdf 
SAINI S, Chaudhary OP, Anoosha, V. 2018. Relationship of population size and extraction frequency with honey production in Apis mellifera colonies. Journal of Entomology and Zoology Studies.6(3):1374-1377. ISSN: 2320-7078. https://www.researchgate.net/profile/Op_Chaudhary2/publication/328902070_Relations hip_of_population_size_and_extraction_frequency_with_honey_production_in_Apis_mel lifera_colonies/links/5bea992d299bf1124fce68f2/Relationship-of-population-size-andextraction-frequency-with-honey-production-in-Apis-mellifera-colonies.pdf

SAS Intitute. 2014. Statistical Analysis Software SAS/STAT®. version 9.4, Cary, N.C., USA: SAS Institute Inc., ISBN: 978-1-60764-599-3. https://www.sas.com/en_us/software/stat.html

SCHNEIDER S, Eisenhardt D, Rademacher E. 2012. Sublethal effects of oxalic acid on Apis mellifera (Hymenoptera: Apidae): changes in behaviour and longevity. Apidologie. $\quad 43(2): \quad 218-225 . \quad$ ISSN: https://doi.org/10.1007/s13592-011-0102-0

SMODIŠ ŠMI, Nakrst M, Žvokelj L, Gregorc A. 2011. The acaricidal effect of flumethrin, oxalic acid and amitraz against Varroa destructor in honey bee (Apis mellifera carnica) colonies. Acta Veterinaria Brno. 80(1): 51-56. ISSN: 1801-7576. https://doi.org/10.2754/avb201180010051

SEMKIW P, Skubida P, Pohorecka K. 2013. The amitraz strips efficacy in control of Varroa destructor after many years application of amitraz in apiaries. Journal of Apicultural Science. 57(1): 107-121. ISSN: 2299-4831. https://content.sciendo.com/view/journals/jas/57/1/article-p107.xml

STRANGE JP, Sheppard WS. 2001. Optimum timing of miticide applications for control of Varroa destructor (Acari: Varroidae) in Apis mellifera (Hymenoptera: Apidae) in Washington State, USA. Entomological Society of America 94(6):1324-1331. ISSN 0022-0493. https://doi.org/10.1603/0022-0493-94.6.1324

VANENGELSDORP D, Meixner MD. 2010. A historical review of managed honey bee populations in Europe and the United States and the factors that may affect them. Journal of Invertebrate Pathology. 103: S80-S95. ISSN: 0022-2011. https://doi.org/10.1016/j.jip.2009.06.011

WILFERT L, Long G, Leggett HC, Schmid-Hempel P, Butlin R, Martin SJM, Boots M. 2016. Deformed wing viruses is a recent global epidemic in honeybees driven by Varroa mites. Research Report. Science. 351(6273):594-597. ISSN 1095-9203. https://doi.org/10.1126/science.aac9976 